\title{
Green Bank Telescope OH Observations of Smith's Cloud: Evidence Of A Lack Of Chemistry
}

\author{
Anthony Minter \\ Green Bank Observatory, National Radio Astronomy Observatory, \\ Green Bank, WV, U.S.A. 24901 \\ email: tminter@nrao.edu
}

\begin{abstract}
Smith's Cloud is a large $f e w \times 10^{6}$ Solar Mass cloud which will impact the Milk Way disk in about 35 Million Years (Lockman et al., 2008). Green Bank Telescope OH observations indicate that there are no molecules present in Smith's Cloud, and thus there is no active ongoing chemistry in Smith's Cloud.
\end{abstract}

Keywords. Keyword1, keyword2, keyword3, etc.

\section{Introduction}

The origin of Smith's Cloud (SC) is uncertain (Lockman et al., 2008; Fox et al., 2016). It could be a primordial cloud, a dwarf galaxy, a dark matter clump accretting gas from the outer disk, or outer disk gas which was launched towards the inner galaxy.

SC impacted the outer Milky Way plane roughly $70 \mathrm{Myr}$ ago and will encounter the inner Milky Way plan in about 35 Myr (Lockman et al., 2008). $H_{\alpha}, N_{I I}$ and $S$ have been detected in SC (Hill et al., 2009; Fox et al., 2016). This indicates that the metalicity of SC is approximately half solar. No stars have been detected in SC (Stark et al., 2015).

Molecules can form whenever $N_{H}>3 \times 10^{20} \mathrm{~cm}^{-2}$ (e.g., see Liszt \& Lucas 2000; Gillmon et al., 2006). SC could have formed molecules in 30-70 Myr (see Liszt \& Lucas 2000). $\mathrm{OH}$ is the simpilest and first molecule to form and is observed in dwarf galaxies but not in HVCs (Barriault et al., 2010).

The Green Bank Telescope was used to limit $\mathrm{OH}$ emmision to $<0.5 \mathrm{mK}$ at a resolution of $1 \mathrm{~km} \mathrm{~s}^{-1}$. This gives a metalicity limit $<3 \%$. and implies that there is no active molecular formation, and thus chemistry, in SC.

The only young, no molecule, no star formation model for the origin of SC is if it is gas from the outer disk of Milky Way (maybe from dark matter passing through disk and accretting disk gas) on a trajectory to the inner disk.

\section{References}

Lockman, F. J., Benjamin, R. A., Heroux, A. H. J., \& Langston, G. I. 2008, ApJL, 679, 21

Fox, A. J., Lehner, N., Lockman, F. J., Wakker, B. P., Hill, A. S., Heitsch, F., Stark, D. V., Barger, K. A., Sembach, K. R., \& Rahman, M. 2016, ApJL, 816, 11

Hill, A. S., Haffner, L. M. \& Reynolds, R. J 2009, ApJL, 703, 1832

Stark, D. V., Baker, A. D. \& Kannappan, S. J. 2015, MNRAS, 446, 1855

Liszt, H. S. \& Lucas, R. 2000, A\&3A, 355, 333

Gillmon, K., Shull, J. M., Tumlinson, J. \& Danforth, C. 2006, ApJ, 636, 891

Barriault, L, Joncas, G., Lockman, F.J. \& Martin, P. G. 2010, MNRAS, 407, 2645 